\title{
Theoretical Analysis and Experimental Realization of Highly Effective Acceptor Ionization in GaN via Mg Co-doped with 4d-Element (In)
}

\author{
Zhiqiang Liu, ${ }^{1, *^{*}}$ Xiaoyan Yi, ${ }^{1,2}$ Junxi Wang, ${ }^{1,2}$ Ian Ferguson, ${ }^{4} \mathrm{Na} \mathrm{Lu}^{3}$ and Jinmin $\mathrm{Li}^{1,2}$
}

In this work, we clarify the underlying physics and the acceptor ionization process in the In-Mg co-doping GaN. The fundamental understandings are also applicable for other co-doping nitride systems. We show the effective acceptors ionization stems mainly from the band structure tuning effect of the $4 \mathrm{~d}$ orbitals of In atoms. In addition, temperature dependent defect-related photoluminescence analysis was proposed to examine the energy position of $\mathrm{Mg}$ impurity levels, which is crucially important in the design for high p-doping. Finally, a hole concentration, which is nearly one order of magnitude higher than what is typically achievable by direct Mg-doping, was achieved.

Keywords: GaN; P-doping; Co-doping; Hole concentration

Received 16 December 2018, Accepted 21 February 2019

DOI: $10.30919 /$ esmm5f209

\section{Introduction}

Obtaining high p-type conductivity in nitrides has been shown to be extremely difficult, and has hindered the development of nitride-based light- emitting devices for decades. Considerable efforts have been made, from the viewpoint of experimental optimization (nonequilibrium growth techniques and high-temperature annealing), to partially address this issue. ${ }^{1-5}$ However, the challenge of p-doping still exists. The underlying physics surrounding the p-doping challenge is still debatable. Furthermore, the lack of a reliable method to analyze the properties of acceptor levels makes p-doping in nitrides more difficult. ${ }^{6}$

Other approaches based on band-engineering have also been developed to achieve effective p-doping in nitrides. Peter and Schubert 7, 8 have found that the low doping efficiency could be partially overcome by polarization induced modulation of the valence band edge in a superlattice. Simon and Jena ${ }^{9}$ also demonstrated that highefficiency p-type doping could be produced using the built-in electronic polarization in bulk uniaxial semiconductor crystals. B. Gunning et al proposed another strategy to lower the acceptor impurity states by extremely high doping and impurity band conduction. ${ }^{10}$ Later, the concept of "impurity resonate states of p-doping" was proposed by Liu et al " ${ }^{11}$. Recently, co-doping has been regarded as another important

'Research and Development Center for Solid State Lighting, Institute of Semiconductors, Chinese Academy of Science, Beijing, 100086, China Center of Materials Science and Optoelectronics Engineering, University of Chinese Academy of Sciences, Beijing 100049, China

'Lyles School of Civil Engineering, Birck Nanotechnology Center, Sustainable Materials and Renewable Technology (SMART) Lab, Purdue University, West Lafayette, IN 47906 USA

${ }^{4}$ Electrical and Computer Engineering, Missouri University of Science and Technology, Rolla MO 65409, USA

*E-mail: lzq@semi.ac.cn strategy to address this issue, ${ }^{1,11-13}$ for example by Si-Mg co-doping and other mutually passivated defect-pair co-dopings. S J Chung et al reported the effect of In co-doping on the properties of p-GaN and revealed the enhancement of PL intensity, which indicated the improvement of p-doping. ${ }^{14}$

In this work, an effective method for achieving efficient p-type doping in high bandgap nitride semiconductors by In-Mg co-doping was analyzed experimentally and theoretically. Compared with previous reports, which are mainly based on experimental observations and from the perspective of suppressing compensation effects, ${ }^{15-18}$ here we concentrate more on the underlying physics and the acceptor ionization process in such co-doping systems.

As well-known, when an impurity with a lower valence electron replaces the host atom in an otherwise perfect semiconductor, an acceptor impurity energy level is introduced. This impurity energy level is at an energy, $E_{A}$, above the valence band maximum (VBM). In terms of Mg-doped nitrides systems, the impurity energy level corresponds to the $2 p$ states of the $\mathrm{Mg}$ atoms. ${ }^{11,13,19,20} E_{A}$, generally called acceptor ionization energy, and refers to the energy difference between the impurity energy level and the VBM of the host. Therefore, it is critically important to study the energy position of $\mathrm{Mg}$ impurity levels related to the VBM of the host for understanding the underlying physics of certain p-doping methods. In this work, this issue was addressed by first principle calculations and temperature dependent defect-related (TDD) photoluminescence (PL) analysis.

\section{Experimental section}

In this study, In-Mg co-doping samples (Sample II) were grown by a Vecco metal organic chemical vapor deposition (MOCVD). Trimethylgallium (TMGa), trimethylindium(TMIn), $\mathrm{CP}_{2} \mathrm{Mg}$ and ammonia were used as precursors for the $\mathrm{p}-\mathrm{GaN}$ growth. The MOCVD chamber pressure was kept at 200 torr during the whole growth process. The growth was initiated on the sapphire substrate with an approximately $20 \mathrm{~nm}$ thick GaN layer. $1 \mu \mathrm{m}$ undoped GaN layers were 
grown subsequently to ensure all electrical measurements resulted only from the p-type $\mathrm{GaN}$. Then, a $0.5 \mu \mathrm{m} \mathrm{In}-\mathrm{Mg}$ co-doping $\mathrm{GaN}$ layer were grown at $1050{ }^{\circ} \mathrm{C}$. A high V/III molar flow ratio of 10000 was used to suppress the formation of a compensation center (nitrogen vacancies). The flow rates of TMGa was fixed to be $18.6 \mathrm{umol} / \mathrm{min}$. While the flow rates of $\mathrm{CP}_{2} \mathrm{Mg}$ was about $0.2 \mathrm{umol} / \mathrm{min}$. TMIn $/ \mathrm{Cp}_{2} \mathrm{Mg}$ molar flow ratio remained at about 5. Conventional $\mathrm{Mg}$-doped $\mathrm{GaN}$ control sample (Sample I) was also prepared using the same growth parameters. All the samples were annealed at $650{ }^{\circ} \mathrm{C}$ for 30 mins in nitrogen to activate the $\mathrm{Mg}$ dopant for growth. Temperature-dependent Hall-effect measurements were performed in a magnetic field of $0.5 \mathrm{~T}$ using the van der Pauw method. NiAu was evaporated as the ohmic contact layer. The TDD PL was characterized using room-temperature

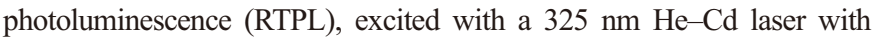
power to be $20 \mathrm{~mW}$. Secondary ion mass spectrometry (SIMS) was performed to check the concentration of dopants.

\section{Results and discussion}

\subsection{Band Structure and Orbital Hybridization Analysis via First} Principle Calculation

Projected densities of states (DOSs) were analyzed to examine the orbital hybridization between doping atoms and the host matrix, using the projector-augmented-wave method with generalized gradient approximations, as implemented in the Vienna ab initio simulation package. $^{21}$

Two Ga atoms are substituted by In and Mg randomly in a $2 \times 2 \times 2$ supercell to examine the electronic structure of In-Mg co-doping In-Mg co-doping. For simplicity, only the most energetically favorable result is presented. We used generalized gradient approximations (GGA) of Perdew-Burke-Ernzerhof (PBE) functionals as the exchange correlation potential. ${ }^{21}$ We choose the cutoff energy as $800 \mathrm{eV}$. All the atoms are fully relaxed to ensure the Hellmann-Feynman force is less than 0.03 eV/ang.

The projected densities of states (DOSs) of In- $\mathrm{t}_{2 d}, \mathrm{~N}-\mathrm{t}_{2 p}$ states and Mg$t_{2 p}$ are shown in Fig. 1. As can be observed, several peaks of the In- $t_{2 d}$ states overlap with that of $\mathrm{N}-\mathrm{t}_{2 p}$, which indicating a strong coupling. ${ }^{22,23}$ As is known, In- $4 d$ states will split into three-fold $t_{2 d}$ states and two-fold $\mathrm{e}_{g}$ states in the environment of the $\mathrm{GaN}$ matrix. Due to the same $\mathrm{t}_{2}$ symmetry, the In- $\mathrm{t}_{2 d}$ and $\mathrm{N}-\mathrm{t}_{2 p}$ states can couple with each other and lead to a strong a level repulsion. ${ }^{2426}$ As a result, the anti-bond states mostly composed of N $2 p$ orbitals will be pushed up to form a "new" VBM. Meanwhile, the bond states mostly composed by $\mathrm{In}_{2 d}$ states will be pushed down and merge with the valence band. The inset shows the isosurface charge density plots of the anti-bond states near the VBM of the co-doping system. It is important to highlight that the charge density is mainly coming from In and surrounding $\mathrm{N}$ atoms. This is a direct evidence to indicate the coupling between In- $t_{2 d}$ and $\mathrm{N}-\mathrm{t}_{2 p}$ states. In Fig. 2, we show the calculated total DOS for pure GaN and In doped GaN,

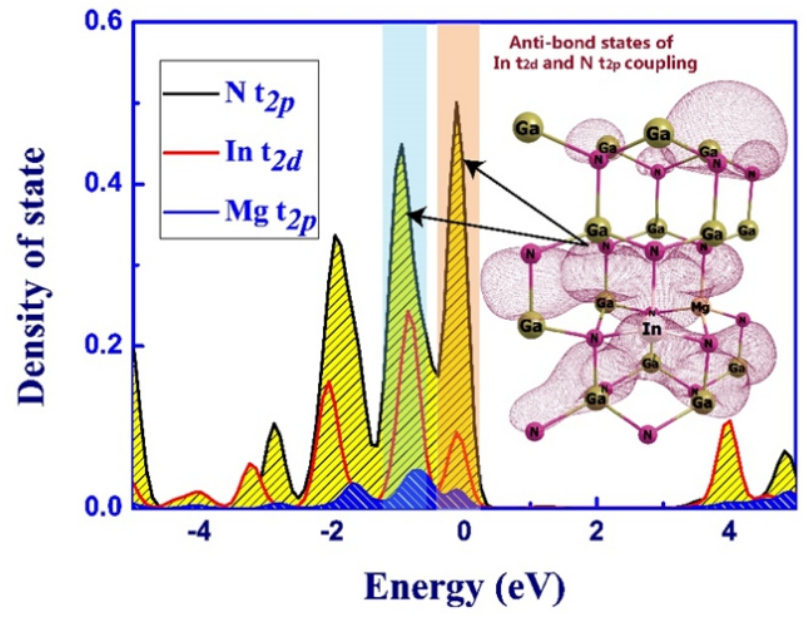

Fig. 1 Calculated projected density of states of In- $4 d, \mathrm{~N}-2 p$ orbitals. The inset shows the isosurface charge density plots of the anti-bond states near the VBM.

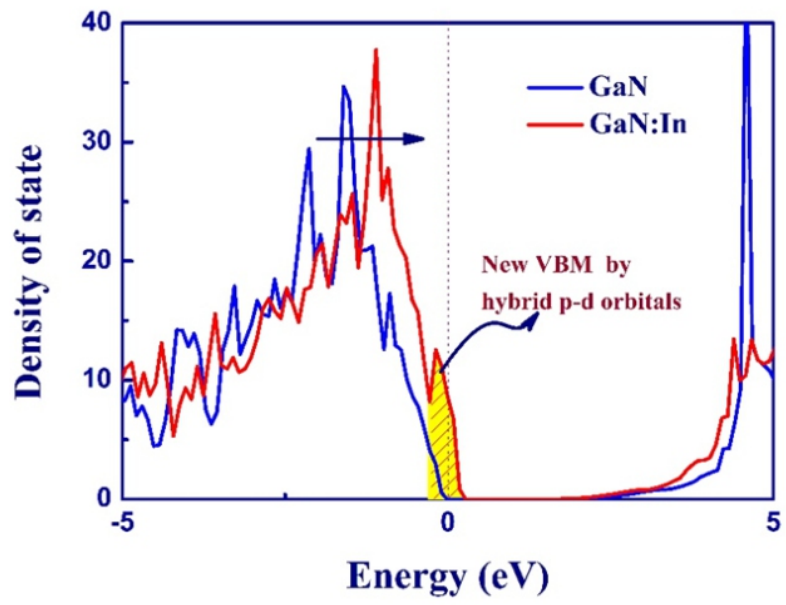

Fig. 2 Calculated total DOS for supercells of pure GaN, and In doped GaN system.

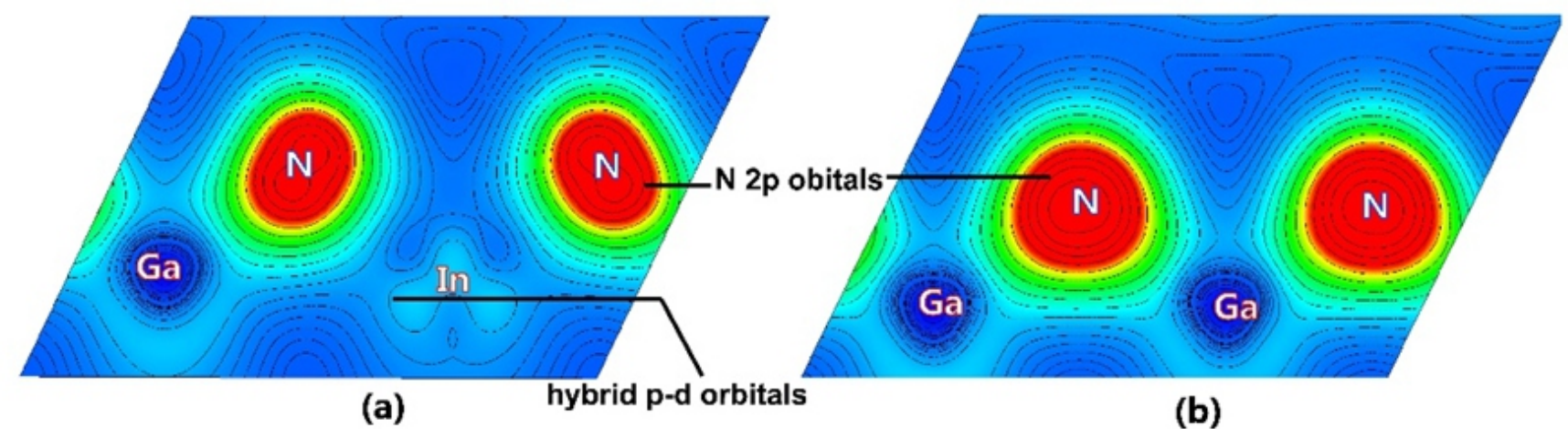

Fig. 3 Isosurface charge density plots of VBM at $\Gamma$ point in the planes of (a) N-In-N bonds and (b) N-Ga-N bonds 
to further clarify the band structure modulation effect of orbital coupling between doping atoms and the host matrix. It is clearly observed that the state distribution of In doped $\mathrm{GaN}$ (blue line) shifts to higher energies. As a result, a "new" higher VBM, which is composed of hybrid In- $\mathrm{t}_{2 d}$ and $\mathrm{N}-\mathrm{t}_{2 p}$ orbitals, is formed. As discussed above, the upward shift of the VBM can facilitate acceptor ionization. Thus better p-type dopability in GaN could be expected. Furthermore, several peaks of $\mathrm{Mg} \mathrm{t} \mathrm{t}_{2 p}$ states also overlap with thoset of $\mathrm{In}_{2 d}$ due to sharing the same symmetry. However, considering the limited atom concentration the direct coupling between In- $\mathrm{t}_{2 d}$ and $\mathrm{Mg}-\mathrm{t}_{2 p}$ state should be considerately weak.

In Fig. 3 we show the calculated charge density of the VBM to further clarify the underlying physics of the above observation. It can be noticed that the VBM states of co-doping system tend to be more localized on the In-N site and exhibits a strong $p-d$ hybridization orbital characteristics. As known, the VBM of InN is $1.11 \mathrm{eV}$ higher than that of $\mathrm{GaN}$ due to larger In-N bond length and strong coupling between $\mathrm{N}$ $2 p$ orbital and In $4 d$ orbitals. ${ }^{27}$ In this scenario, the VBM turns to be more InN-like and higher than pure GaN. Therefore, the In doping system turn to be more easier to be p type doped. It is worthwhile mentioning that the limited concentration of Indium atoms (only about $10^{17} / \mathrm{cm}^{-3}$ confirmed by secondary ion mass spectrometry, not shown here), does not significantly change band-gap. That means Indium atoms still acts as dopant. Subsequently, it will be further proved by the PL spectrum.

\subsection{Impurity States Characterization via TDD PL Analysis}

To understand the acceptor ionization process of the In-Mg co-doping system, next, a phenomenological rate-equation model was proposed to analyze the temperature dependence of the defect-related photoluminescence (PL) of In-Mg co-doped GaN samples, and taken as a diagnostic technique to examine the energy position of the $\mathrm{Mg}$ impurity (acceptor) level.

The intensity of the PL emission typically decreases monotonically with increasing temperature. As known, the underlying physical mechanism of such PL emission-quenching is the redistribution of carriers into different energy levels (i.e. impurity levels, conduction band minimum, or valence band maximum of the host material) following the excitation. These processes dominate the competition between different recombination channels. Therefore, the PL intensity of a specific band can be used to determine the characteristics of given point defects/impurities and carrier transitions in semiconductors. ${ }^{28,29}$

Generally, the room temperature band-edge photoluminescence emission of pure $\mathrm{GaN}$ is observed at about $3.4 \mathrm{eV}$. However, for p-type $\mathrm{GaN}$ it is well established that the Ultraviolet Luminescence (UVL) band (from $3.1 \mathrm{eV}$ to $3.35 \mathrm{eV}$ ) followed by a few LO photon replicas is dominated for low and moderate $\mathrm{Mg}$ doping $\mathrm{GaN}{ }^{30}$ While for high $\mathrm{Mg}$ doping sample, the peak of UVL apparently transform to a broad Blue Luminescence (BL) band (from $2.2 \mathrm{eV}$ to $3.1 \mathrm{eV}$ ). It was also noted that the emergence of BL band indicated the self-compensation. In another word, by further increasing dopant concentration, the hole concentration will decrease ${ }^{31}$. Next, based on the TDD PL measurement, the characteristics of given point defects/impurities and carrier transitions was analyzed.

Fig. 4 shows the evolution of the PL spectrum of sample I and sample II at selected temperatures ranging from $20 \mathrm{~K}$ to $300 \mathrm{~K}$. Two sets of the defect-related PL bands can be clearly resolved by Gaussian fitting, $U V L$ band (from $3.1 \mathrm{eV}$ to $3.35 \mathrm{eV}$ ) with the main peak at $3.3 \mathrm{eV}$ and its LO phonon replicas and the $B L$ band (from $2.2 \mathrm{eV}$ to $3.1 \mathrm{eV}$ ) with the main peak at $2.9 \mathrm{eV}$, which is attributed to nitrogen vacancy $\left(\mathrm{V}_{\mathrm{N}}\right)$ related deep donors. ${ }^{32,33}$ To gain a better view, the fitting processes are not shown in Figure 4. The $U V L$ band originates from the shallow donor-acceptor pair $(D A P)$ and/or conduction-band-acceptor $(e-A)$ transition. $^{30,34,35}$ The shapes of the DAP and $e-A$ bands are similar due to sharing the same acceptor level and relatively small donor ionization energy. Therefore, they cannot always be discriminated from each other. $^{30,36,37}$ The $U V L$ band dominates at low temperatures, and decreases slowly as the temperature increases from $10 \mathrm{~K}$ to about $80 \mathrm{~K}$, then exhibits an abrupt quenching in the temperature range of $80 \mathrm{~K}$ to $180 \mathrm{~K}$. Further increasing the temperatures, the BL band becomes dominated and the $U V L$ band disappears under the tail of the BL band. ${ }^{34,38} \mathrm{As}$ shown in Fig. 5, the total $U V L$ band intensity is decreased more than two orders of magnitude in the temperature range of $20 \mathrm{~K}-300 \mathrm{~K}$. It is important to notice that the characteristic temperature $T_{0}$, at which the abrupt thermal quenching begins, decreases as In incorporation takes place. For instance, the $U V L$ band from sample I can still be resolved at
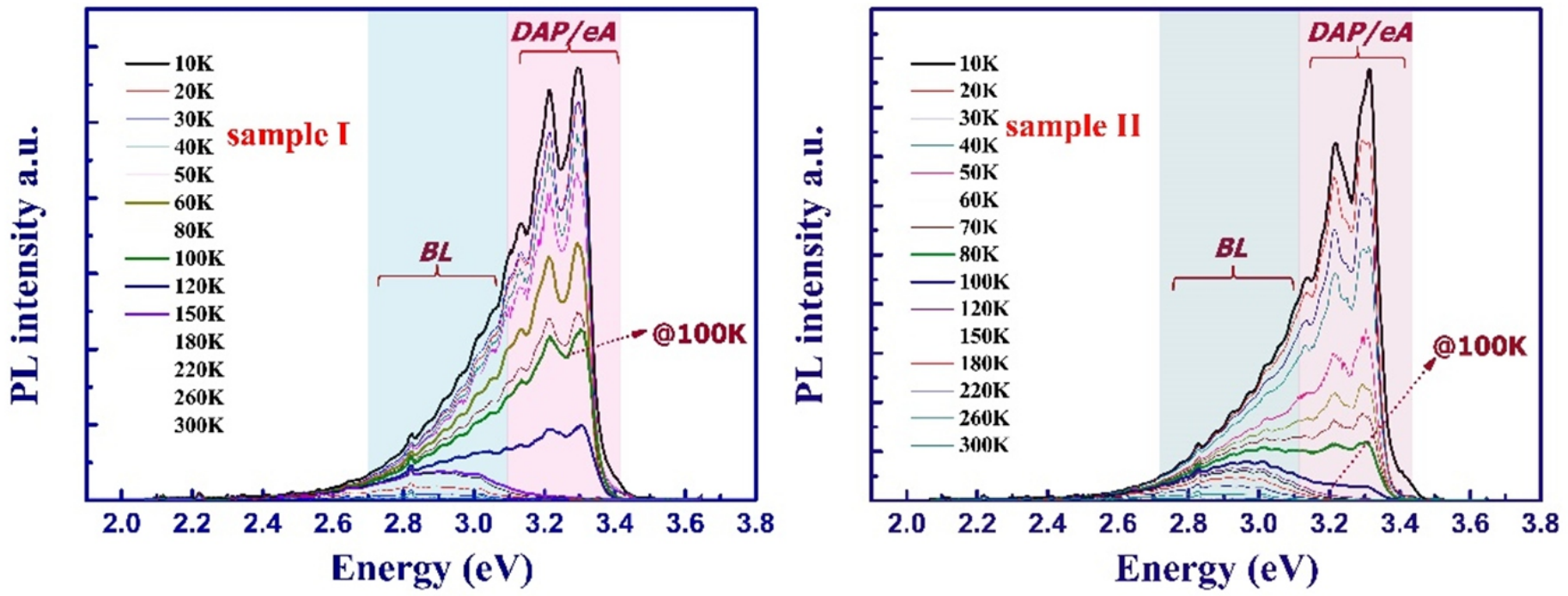

Fig. 4 Evolution of PL spectrum in Mg doped (sample I ) and In-Mg codoped (sample II ) GaN with increasing temperature. The characteristic temperature $T_{0}$, at which the abrupt thermal quenching begins, seem to decreases with In incorporation. 
$100 \mathrm{~K}$. However, the $U V L$ band from sample II could not be detected. Next, we will show that such decreasing of the characteristic temperature $T_{0}$ indicates the $\mathrm{Mg}$ acceptor levels are more adjacent to the VBM of the host. In other words, the Mg acceptors are more easier to be ionized.

As shown in Fig. 6, the minimal impurities participating in carrier distribution and recombination should include donor, acceptor and deep center (either radiative or nonradiative) types.

Taking into account all the possible processes, the kinetic equations under steady-state conditions for the conduction band and the donor levels are given by

$$
\begin{aligned}
& \frac{\partial n}{\partial t}=G-C_{n d} N_{D} n-C_{n s} N_{s} n-C_{n a} P_{A}^{0} n+N_{D}^{0} T_{n} \exp \left(-E_{n} / k T\right)=0, \\
& \quad \text { and } \\
& \frac{\partial N_{D}^{0}}{\partial t}=C_{n d} N_{D} n-C_{\mathrm{da}} P_{A}^{0} N_{D}^{0}-N_{D}^{0} T_{n} \exp \left(-E_{n} / k T\right)=0,
\end{aligned}
$$

In Eq. (1), the first term is the inter-band generation rate. The second term describes the electrons captured by the donor levels. The third term refers to the loss of "non-radiative" recombination via the deep states. Note that the recombination loss at the deep centers can be either radiative or nonradiative, including "BL". We refer to all these recombination channels as "non-radiative", as far as the band edge radiative recombination is concerned. The fourth term is the free-tobound recombination, and the fifth term is the process of electron ionization back to CB. The exciton peak cannot be resolved in our PL spectrum, even at low temperature. Therefore, it is reasonable to ignore the contribution from the excitonic transition.

Similarly, the second term in Eq. (2) is the radiative recombination of the donor-bound electrons. Here, $\mathrm{N}_{\mathrm{D}}$ and $\mathrm{N}_{\mathrm{A}}$ are the n-type and p-type doping concentration, respectively. In p-type systems at low excitation intensity, $N_{D}{ }^{0}<<N_{D}$, ${ }^{30}$ the rate of the free electrons captured by donors after excitation could be expressed as $C_{n d}\left(N_{D}-N_{D}^{0}\right) n \approx C_{n d} N_{D} n$, where $C_{n d}$ is the electron-capture coefficient for the donors, $N_{D}{ }^{0}$ is the concentration of electrons at donor levels (neutral donors), and $n$ is the electron concentration of conduction band (CB). Here, $C_{n a}$ is the electron-capture coefficient for the acceptor, and $P_{A}^{0}$ is the hole concentration at acceptor levels (neutral or non-ionized acceptors). $C_{n s}$ is the electron capture coefficient for the deep center, and $N_{\mathrm{s}}$ is the concentration of the deep centers. At elevated temperatures, the bound electrons at donors may return to the conduction band as a result of thermal activation. The probability of this process is proportional to $T_{n}$, $\exp \left(-E_{n} / k T\right)$, where $E_{n}$ refer to the thermal activation energy for the donors, $k$ and $T$ are the Boltzmann constant and temperature, respectively, $T_{\mathrm{n}}$ is a constant, and $C_{d a}$ is the donor-bound electron-capture coefficient for the acceptor.

The total UVL can be calculated as

$$
I^{P L}=C_{d a} \cdot N_{D}^{0} \cdot P_{A}^{0}+C_{n a} \cdot n \cdot P_{A}^{0}
$$

Next, we consider the hole transitions. At a low excitation condition, in a p-type material, the hole concentration of the acceptor level is much higher the than for the excited electrons, and therefore can be approximately expressed by the thermal distribution

$$
P_{A}^{0}=N_{A} /\left(1+A \exp \left(-E_{p} / k T\right)\right.
$$

where $\mathrm{A}$ is a constant, and $E_{\mathrm{p}}$ is the acceptor binding energy.

As we previously reported, ${ }^{6}$ by solving Eq. (1-4) the values of $E_{p}$ and $E_{n}$ can be obtained by iteratively fitting the PL intensity as a function of temperature, and shown in Fig. 5. The acceptor ionization energy are about $237.0 \mathrm{meV}$ for the reference sample II, and $87.5 \mathrm{meV}$ for the In-Mg co-doping sample $I$. As mentioned, the acceptor ionization energy can also be understood as the energy position of the $\mathrm{Mg}$ acceptor level related to the VBM of the host, as schematically shown in Fig.7. This is further evidence to support our first-principle calculation results. The upward shift of the VBM facilitates acceptor activation and thus better p-type dopability in GaN.

\subsection{Temperature Dependenc Hall And Sims Measruement}

Temperature dependence Hall measurement was performed to further test the results of our TDD PL analysis, as shown in Fig. 8. The measured hole concentration for reference sample II was approximately $1.55 \times 10^{17} \mathrm{~cm}^{-3}$. As In incorporation increased, the hole concentration for sample II approaches to be $1.94 \times 10^{17} \mathrm{~cm}^{-3}$. The acceptor activation was

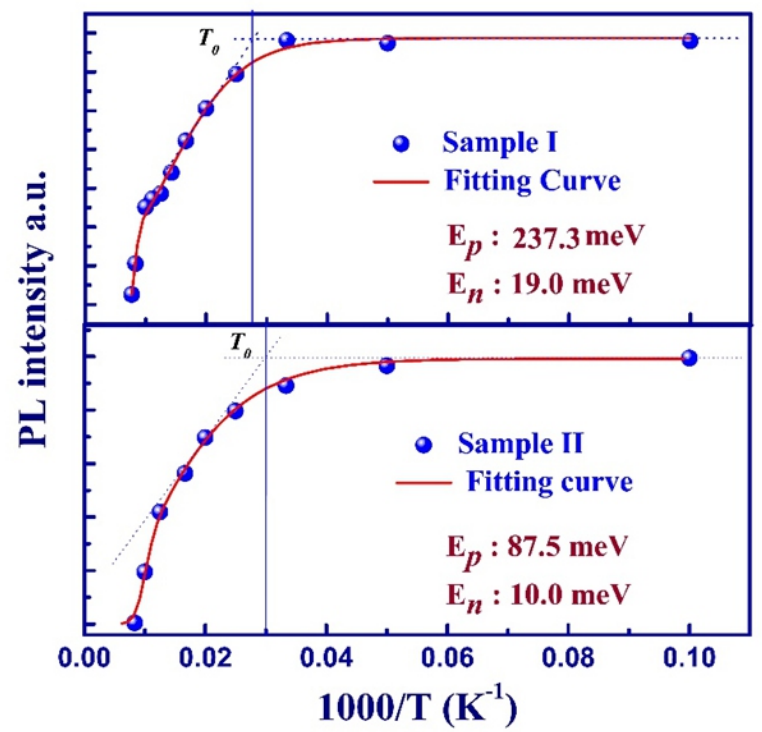

Fig. 5 Temperature dependence of the PL intensity of UVL band. The ionization energies are shown. The fitted curves are shown as solid lines.

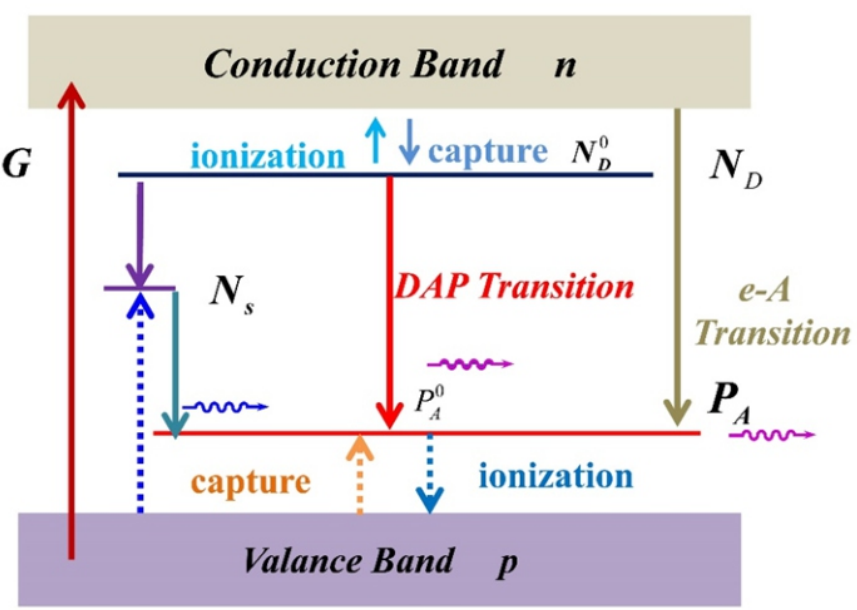

Fig. 6 Band diagram and the main transitions after excitation. Transitions of electrons and holes are shown with solid and dashed arrows, respectively. In this work, three types of defects, shallow donors, shallow acceptors, deep nonradiative centers with concentration $N_{D}, P_{A}, N_{s}$, were considered. 
determined by fitting the concentration data, as shown in Fig.8, and was consistent with the results from our TDD PL analysis. As shown in Figure 9, secondary ion mass spectrometry measurements were performed to verify the incorporation and distribution of $\mathrm{Mg}$ and $\mathrm{In}$ atoms. The concentration of $\mathrm{In}$ and $\mathrm{Mg}$ is about $1 \times 1020 \mathrm{~cm}^{-3}$ and $1 \times 1017 \mathrm{~cm}^{-3}$, respectively. Due to the limited concentration, both of them still act as dopant in the GaN matrix, not alloy.

\section{Conclusions}

To conclude, effective acceptor ionization in $4 d$ Element (In)-Mg codoping GaN Systems has been achieved experimentally and the underlying physics and the specific acceptor ionization process has been analyzed theoretically. A hole concentration as high as $1.94 \times 10^{18} \mathrm{~cm}^{-3}$, which is nearly one order of magnitude higher than typical value obtained through direct $\mathrm{Mg}$-doping, has been achieved in In-Mg codoping GaN. The elevating of the VBM $(\sim 150 \mathrm{meV})$ facilitates acceptor activation and thus better p-type dopability in $\mathrm{GaN}$, as convinced from the first-principle calculation and a diagnostic technique using thermal quenching analysis. Our reports show significance on the p-doping mechanism of $\mathrm{GaN}$ and will advance the development of $\mathrm{GaN}$ based optoelectronic and electronic device.

\section{Conflict of interest}

"There are no conflicts to declare".

\section{Acknowledgements}

Financial supports from National Key R\&D Program of China (Grant No. 2017YFB0403300 2017YFB0403302), and the National Key

Research and Development Program of China (2016YFB0400102).

\section{References}

1. Y. Aoyagi, M. Takeuchi, S. Iwai and H. Hirayama, Appl. Phys. Lett., 2011, 99, 112110.

2. K. S. Kim, M. S. Han, G. M. Yang, C. J. Youn, H. J. Lee, H. K. Cho and J. Y. Lee, Appl. Phys. Lett., 2000, 77, 1123-1125.

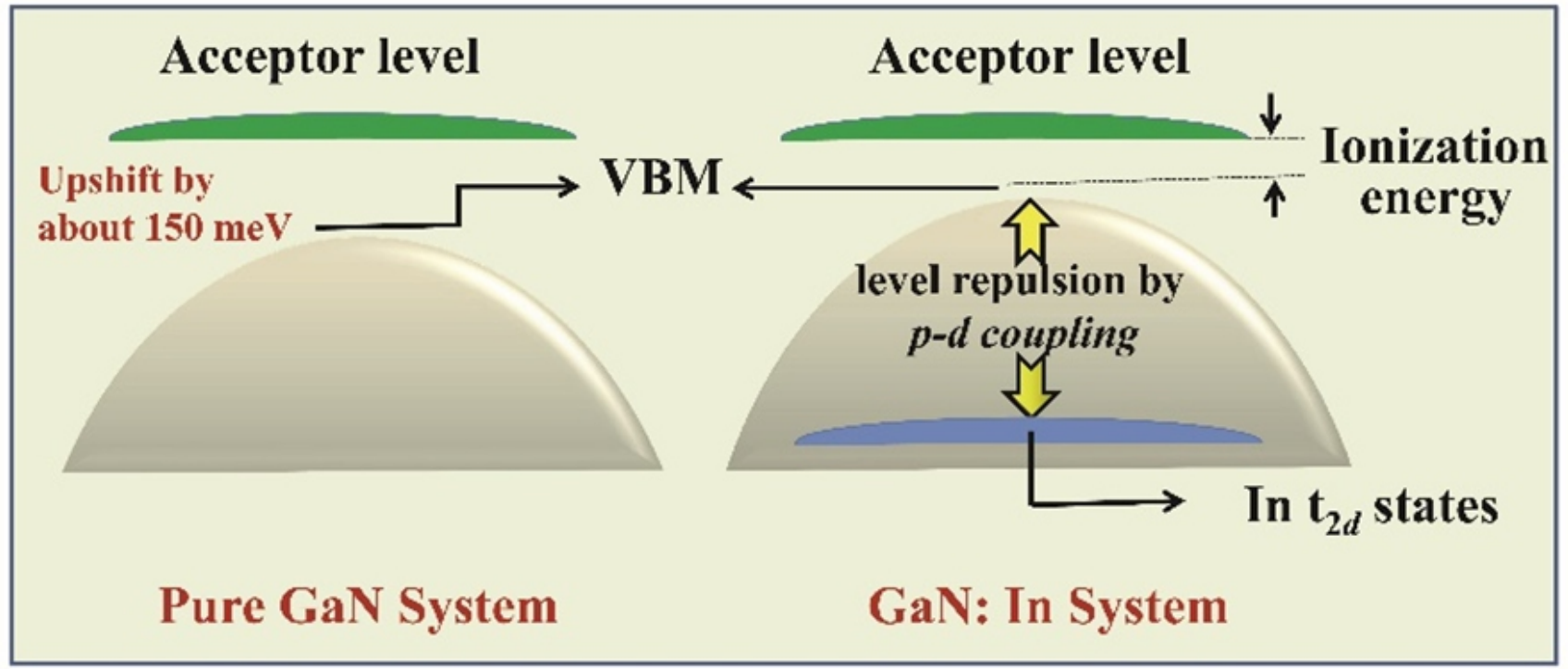

Fig. 7 Schematic model showing the position and the hybridization and level repulsions between $\mathrm{In}_{2 d}$ and $\mathrm{N}_{2 p}$ states.

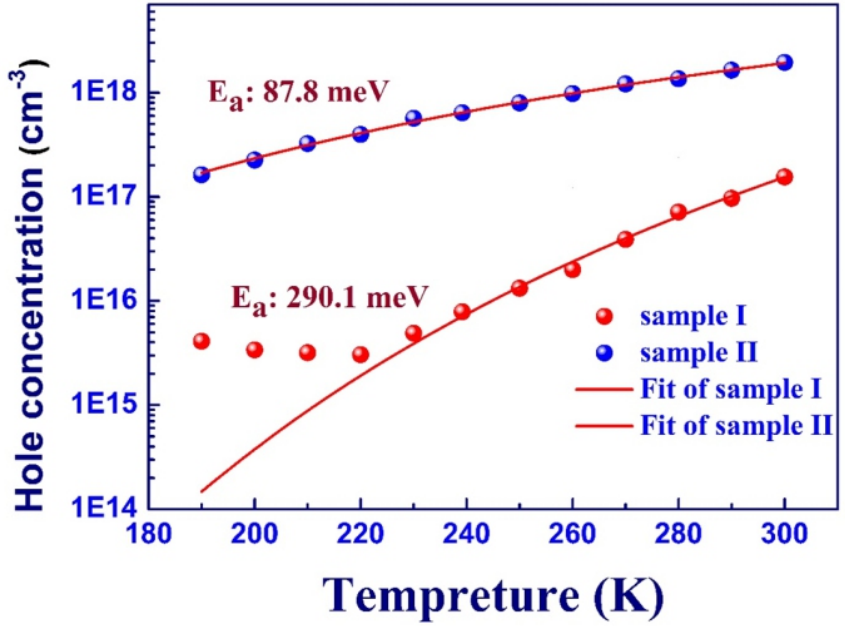

Fig. 8 Temperature dependence hole concentration for In-Mg co-doping GaN samples. The fitted curve is shown with a solid line.

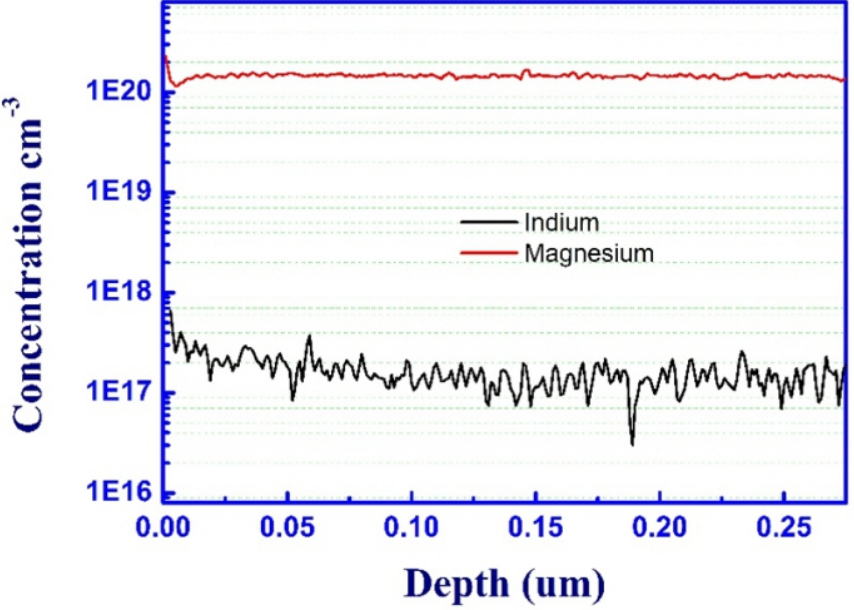

Fig. 9 Secondary ion mass spectrometry measurements were performed to check the concentration of $\mathrm{Mg}$ and In atoms. 
3. T. Tanaka, A. Watanabe, H. Amano, Y. Kobayashi, I. Akasaki, S. Yamazaki and M. Koike, Appl. Phys. Lett., 1994, 65, 593-594.

4. Y. F. Yan, J. B. Li, S. H. Wei and M. M. Al-Jassim, Phys. Rev. Lett., 2007, 98, 135506.

5. S. Nakamura, Science, 1998, 281, 956-961.

6. Z. Liu, Y. Huang, X. Yi, B. Fu, G. Yuan, J. Wang, J. Li and Y. Zhang, Sci. Rep., 2016, 6, 32033

7. E. F. Schubert, W. Grieshaber and I. D. Goepfert, Appl. Phys. Lett., 1996, 69, 3737-3739.

8. P. Kozodoy, Y. P. Smorchkova, M. Hansen, H. L. Xing, S. P. DenBaars, U. K. Mishra, A. W. Saxler, R. Perrin and W. C. Mitchel, Appl. Phys. Lett., 1999, 75, 2444-2446.

9. J. Simon, V. Protasenko, C. X. Lian, H. L. Xing and D. Jena, Science, 2010, 327, 60-64.

10. B. Gunning, J. Lowder, M. Moseley and W. A. Doolittle, Appl. Phys. Lett., 2012, 101, 082106

11. Z. Q. Liu, X. Y. Yi, Z. G. Yu, G. D. Yuan, Y. Liu, J. X. Wang, J. M. Li, N. Lu, I. Ferguson and Y. Zhang, Sci. Rep., 2016, 6, 23950.

12. Y. Q. Gai, J. B. Li, S. S. Li, J. B. Xia, Y. F. Yan and S. H. Wei, Physical Review B, 2009, 80, 153201.

13. Z. Q. Liu, B. L. Fu, X. Y. Yi, G. D. Yuan, J. X. Wang, J. M. Li, Luna and I. Ferguson, Rsc Advances, 2016, 6, 5111-5115.

14. S. J. Chung, M. S. Kumar, Y. S. Lee, E. K. Suh and M. H. An, Journal of Physics D-Applied Physics, 2010, 43.

15. C. K. Shu, J. Ou, H. C. Lin, W. K. Chen and M. C. Lee, Appl. Phys. Lett., 1998, 73, 641-643.

16. H. M. Chung, W. C. Chuang, Y. C. Pan, C. C. Tsai, M. C. Lee, W. H. Chen, W. K. Chen, C. I. Chiang, C. H. Lin and H. Chang, Appl. Phys. Lett., 2000, 76, 897-899.

17. F. C. Chang, W. C. Chou, W. H. Chen, M. C. Lee, W. K. Chen and H. Y. Huang, Jap. J. Appl. Phys., 2005, 44, 7504-7506.

18. Y. D. Chen, H. L. Wu, G. L. Yue, Z. M. Chen, Z. Y. Zheng, Z. S. Wu, G. Wang and H. Jiang, Phys. Status Solidi B, 2015, 252, 1109-1115.

19. Y. Zhang and J. W. Wang, Phys. Rev. B, 2014, 90, 155201.

20. Z. Q. Liu, A. G. Melton, X. Y. Yi, J. W. Wang, B. Kucukgok, J. Kang, N. Lu,
J. X. Wang, J. M. Li and I. Ferguson, Applied Physics Express, 2013, 6 042104

21. G. Kresse and J. Furthmuller, Phys. Rev. B, 1996, 54, 11169-11186.

22. L. Liu, P. Y. Yu, Z. X. Ma and S. S. Mao, Phys. Rev. Lett., 2008, 100, 127203.

23. G. M. Dalpian and S. H. Wei, Physical Review B, 2005, 72, 115201.

24. G. M. Dalpian, S. H. Wei, X. G. Gong, A. J. R. da Silva and A. Fazzio, Solid State Commun., 2006, 138, 353-358.

25. A. Franceschetti, S. V. Dudiy, S. V. Barabash, A. Zunger, J. Xu and M. van Schilfgaarde, Phys. Rev. Lett., 2006, 97.

26. Y. J. Zhao, P. Mahadevan and A. Zunger, J. Appl. Phys., 2005, 98.

27. F. Wang, J. B. Li, S. S. Li, J. B. Xia and S. H. Wei, Phys. Rev. B, 2008, 77, 113202.

28. M. A. Reshchikov, A. A. Kvasov, M. F. Bishop, T. McMullen, A. Usikov, V. Soukhoveev and V. A. Dmitriev, Phys. Rev. B, 2011, 84, 075212.

29. M. A. Reshchikov and R. Y. Korotkov, Phys. Rev. B, 2001, 64, 115205.

30. M. A. Reshchikov and H. Morkoc, J. Appl. Phys., 2005, 97, 061301.

31. H. Obloh, K. H. Bachem, U. Kaufmann, M. Kunzer, M. Maier, A. Ramakrishnan and P. Schlotter, J. Cryst. Growth, 1998, 195, 270-273.

32. A. F. Wright, C. H. Seager, S. M. Myers, D. D. Koleske and A. A. Allerman, J. Appl. Phys., 2003, 94, 2311-2318.

33. P. J. Hansen, Y. E. Strausser, A. N. Erickson, E. J. Tarsa, P. Kozodoy, E. G. Brazel, J. P. Ibbetson, U. Mishra, V. Narayanamurti, S. P. DenBaars and J. S. Speck, Appl. Phys. Lett., 1998, 72, 2247-2249.

34. A. K. Viswanath, E. J. Shin, J. I. Lee, S. Yu, D. Kim, B. Kim, Y. Choi and C. H. Hong, J. Appl. Phys., 1998, 83, 2272-2275.

35. J. K. Sheu, Y. K. Su, G. C. Chi, B. J. Pong, C. Y. Chen, C. N. Huang and W. C. Chen, J. Appl. Phys., 1998, 84, 4590-4594.

36. M. A. Reshchikov, F. Shahedipour, R. Y. Korotkov, B. W. Wessels and M. P. Ulmer, J. Appl. Phys., 2000, 87, 3351-3354.

37. C. G. Van de Walle, Phys. Rev. B, 1997, 56, 10020-10023.

38. M. Leroux, N. Grandjean, B. Beaumont, G. Nataf, F. Semond, J. Massies and P. Gibart, J. Appl. Phys., 1999, 86, 3721-3728.

Publisher's Note Engineered Science Publisher remains neutral with regard to jurisdictional claims in published maps and institutional affiliations. 